Total saponins isolated from Radix et Rhizoma Leonticis suppresses tumor cells growth by regulation of PI3K/Akt/mTOR and p38

\title{
MAPK pathways
}

Authors: Yingzhuan Zhan, Rui Liu, Wenjie Wang, Jing Li, Xiaoyan Ou Yang,

Yanmin Zhang*

Authors' Affiliations: School of Pharmacy, Health Science Center, Xi'an Jiaotong

University, No. 76, Yanta Weststreet, \#54, Xi’an, Shaanxi Province 710061, P.R.

China

Corresponding Author: Yanmin Zhang, School of Pharmacy, Health Science Center,

Xi'an Jiaotong University, No. 76, Yanta Weststreet, \#54, Xi'an, Shaanxi Province

710061, P.R. China.

Phone: +86 29 82656264; fax: +86 2982655451 .

E-mail: zhang2008@mail.xjtu.edu.cn 
Abstrtact: Both PI3K/AKT/mTOR and mitogen activated protein kinase (MAPK) signaling cascades played an important role in tumorigenesis, a more complete understanding of these signaling pathways allowed the development of new therapeutic strategies. Total saponins isolated from Radix et Rhizoma Leonticis (RLTS) was recognized with anticancer properties. In a murine hepatocellular carcinoma H22 cell-bearing mouse model, RLTS exhibited significant inhibitory effect on tumor growth. Here, we investigated the role of RLTS on the PI3K/AKT/mTOR signaling pathway and MAPK pathways in liver and lung cancer cells. Results obtained showed RLTS inhibited cell proliferation and induced cell apoptosis in vitro, which attributed to the inhibition on the activation of $\mathrm{PI} 3 \mathrm{~K} / \mathrm{AKT} / \mathrm{mTOR}$ cascade and its related signaling molecules, such as activated VEGFR and NF- $\kappa \mathrm{B}$, and activation of p38 MAPK in tumor cells. Additional, RLTS inhibited cell migration and downregulated proteins that mediated metastasis including CXCR4, MMP2 and MMP9. Overall, these findings suggested that RLTS interfered with multiple signaling cascades involved in tumorigenesis and had potential in cancer therapy.

Key words: Total saponins, hepatocellular carcinoma, lung cancer, PI3K/Akt/mTOR, p38 MAPK

\section{Introduction}

The phosphatidylinositol 3-kinase (PI3K)-protein kinase B (PKB/AKT)-mammalian target of rapamycin (mTOR) kinase cascade (PI3K/AKT/mTOR) is a signal transduction pathway activated in the majority of human cancers. It is involved in the regulation of multiple cellular functions including cell proliferation, survival, differentiation, adhesion, motility and invasion (Zhang et al.,2013; Gil, 2014; Slomovitz and Coleman, 2012). The pathway is switched on through activation of 
membrane receptors, including tyrosine-kinase receptors (RTKs), such as VEGFR and PDGFR (Slomovitz and Coleman, 2012; Trinh XB et al., 2009; Zhang et al., 2003). Also, activation of this pathway in tumor cells can increase VEGF secretion by both HIF-1 dependent or independent mechanisms, and regulate angiogenesis by modulating expression of nitric oxide and angiopoietins (Karar and Maity, 2011). The potential of $\mathrm{PI} 3 \mathrm{~K} / \mathrm{AKT} / \mathrm{mTOR}$ pathway inhibition to enhance clinical response of cancer therapy is currently the focus of many preclinical and clinical studies. Many agents have been developed targeting AKT and/or mTOR signaling in tumor cells, and these drugs have effects on tumor cell proliferation and survival.

Besides the PI3K/AKT/mTOR pathways, mitogen-activated protein kinases (MAPKs) are serine-threonine protein kinases that play an important role in the regulation of many cellular processes including cell growth and proliferation, differentiation, and apoptosis. ERk1/2 MAPKs, Jun N-terminal kinase (JNK) and p38 MAPK pathways are three major mammalian MAPK pathways (Wagner and Nebreda, 2009). Especially, p38 MAPK is a tumor suppressor nuclear protein, and regulate cell cycle progression at different transition points by both transcription dependent and transcription-independent mechanisms (Bradham and McClay, 2006). Radix et Rhizoma Leonticis ("Hong Mao Qi" in Chinese) is a plant that widely distributes in China. Previous studies demonstrated that taspine isolated from the roots of Radix et Rhizoma Leonticis had many pharmacological actions such as bacteriostasis, antibiosis, antivirus, anti-inflammatory, anti-ulcer and anti-cancer effects (Zhang et al., 2008). Saponins are a class of chemical compounds found in nature plants, which are considered as bioactive constituents in many plants due to their various pharmacological effects. Total saponins were isolated successfully from Radix et Rhizoma Leonticis (RLTS) in our laboratory. In this study, we characterized 
the anti-cancer effects of RLTS in vivo and assessed PI3K/AKT/ mTOR signaling decades and p38 MAPK involved in anti-proliferative effects of RLTS in cancer cells.

\section{Materials and methods}

\subsection{Animals and cell culture}

4-6 weeks Kunming SPF mice (body wt. 18-22 g) provided by Animal Research Center of Xi'an Jiao tong University (Xi'an, China) were used for all experiments. The methods were carried out in accordance with the approved guidelines of the regional authorities according to Xi'an Jiao tong University animal-care regulations. Hepatocellular carcinoma cell lines including HepG2, BEL-7402 and SMMC-7721, and lung cancer cell lines including A549, NCI-H1299 and NCI-H460 were obtained from Shanghai Institute of Cell Biology in the Chinese Academy of Sciences. BEL-7402, SMMC-7721, A549, NCI-H1299 and NCI-H460 cells were maintained in RPMI1640 medium (Sigma-Aldrich, USA) supplemented with 10\% (v/v) fetal bovine serum (FBS) (Hyclone, China), HepG2 cells and L-02 cells were cultured in Dulbecco’s Minimal Essential Medium (DMEM) (Sigma-Aldrich, USA) supplemented with $10 \%(\mathrm{v} / \mathrm{v}) \mathrm{FBS}$. All cell lines were incubated at $37^{\circ} \mathrm{C}$ in a $5 \% \mathrm{CO}_{2}$ incubator with saturated humidity.

\subsection{Extraction and purification of RLTS}

Radix et Rhizoma Leonticis used in the study was purchased from the TCM Store (Xi'an, P.R. China) and was grounded to 40 mesh. The raw material was extracted once with 8 volumes of $90 \%$ ethanol for $2 \mathrm{~h}$ and then $80 \%$ ethanol by refluxing for $2 \mathrm{~h}$ twice. The ethanol water solution was condensed to an extractum. The extractum was percolated twice with 12 volumes of $1 \% \mathrm{HCl}$ for $30 \mathrm{~h}$. The merged supernatant was concentrated, the $\mathrm{pH}$ value was adjusted to $3-5$. The percolate was obtained by treating the concentrate with LSD001 ion exchange resin, and was chromatographed 
over D101 resin column eluted with 95\% ethanol. Total saponins (RLTS) was gotten by evaporating the eluent under reduced pressure and dried in vacuum conditions overnight. RLTS was dissolved in dimethyl sulfoxide (DMSO) and diluted to the desired concentrations before use. The concentration of DMSO was kept below $0.1 \%$ $(\mathrm{v} / \mathrm{v})$ in the treated groups. $0.1 \%(\mathrm{v} / \mathrm{v})$ DMSO was used as a vehicle control throughout the study.

\subsection{Animal experiments}

Mice housed under aseptic and ventilated condition were inoculated by subcutaneous injection into the oxter of mice with $\mathrm{H} 22$ cells resuspended in $5 \%$ saline. Tumor size was measured on alternate days by using a vernier caliper and calculated by length $x$ width ${ }^{2} \times 0.5\left(\right.$ in $\left.\mathrm{cm}^{3}\right)$. Body weights were monitored weekly as an indicator of overall health. Treatment began when tumors were palpable and no less than $0.1 \mathrm{~cm}^{3}$ in volume. The mice were randomly assigned to control and treatment groups $(n=8$ per group). Animals were administrated orally with RLTS daily at doses of $100 \mathrm{mg} / \mathrm{kg}$ while control animals received equivalent volumes of dissolvant. After a total of 14 days of treatment, mice were sacrificed and tumor tissue was removed and weighed. The anticancer activity in vivo was expressed as an inhibitory rate calculated by the formula: $[(\mathrm{A}-\mathrm{B}) / \mathrm{A}] \times 100 \%$, where $\mathrm{A}$ and $\mathrm{B}$ were the mean tumor weights of the control and treatment groups, respectively.

\subsection{Cell viability assay}

HepG2, BEL-7402, SMMC-7721, A549, NCI-H1299, NCI-H460 and L-02 cells were cultured in 96-well plates, and fresh medium with or without RLTS, was added for 48 h. Cell proliferation reagent MTT (Sigma-Aldrich, USA) was added and incubated at $37{ }^{\circ} \mathrm{C}$ and $5 \% \mathrm{CO}_{2}$ for $4 \mathrm{~h}$. Absorbance was then measured at $490 \mathrm{~nm}$ with a microplate reader (Bio-RAD instruments, USA). 


\subsection{Colony formation assay}

SMMC-7721 and NCI-H460 cells were cultured in 6-well plates and fresh medium with or without RLTS for 10-15 days. Colonies with cell numbers of $>50$ cells per colony were counted after staining with $0.2 \%$ crystal violet (Beijing Chemical Works, China) solution. All the experiments were performed in triplicate wells in three independent experiments.

2.6 Wound healing assay

SMMC-7721 and NCI-H460 cells were planted into 6-well plate and allowed to grow to $70 \%$ confluency in complete medium. Cells were then serum starved for $24 \mathrm{~h}$, and cell monolayers were scratched with a pipette tip. Wounded monolayers were then washed several times with serum-free medium to remove floating cells and photographed in microscope. Cells were incubated in medium in the absence or presence of RLTS for $24 \mathrm{~h}$, cell migrating into the wound surface and the average distance of migrating cells was determined under an inverted microscope.

2.7 Analyses of the cell apoptosis

NCI-H460 cells were plated in culture flask and incubated for $24 \mathrm{~h}$ after the medium was changed to serum-free medium. Cells were treated with RLTS for $48 \mathrm{~h}$, and then trypsinized, collected, washed in PBS, and incubated in $5 \mu \mathrm{L}$ Annexin V-FITC, $10 \mu \mathrm{L}$ PI for 15 min away from light. Samples were analyzed on the flow cytometer (BD Biosciences, USA).

\subsection{Immunoblotting analysis}

SMMC-7721 and NCI-H460 cells were plated in culture flask and treated with RLTS at different concentrations for $48 \mathrm{~h}$. Cells were then harvested and lysed in RIPA Lysis Buffer (Applygen Technologies, China) supplemented with protease inhibitor cocktail tablets and phosphatase inhibitor cocktail tablets (Roche, Switzerland). The 
cell lysates were centrifuged at $12,000 \times \mathrm{g}$ at $4{ }^{\circ} \mathrm{C}$ for 10 minutes. Protein concentration of the supernatants was determined by a protein assay kit (Bio-Rad Laboratories, USA). Equivalent amount of protein was resolved by 10\% SDS-PAGE and transferred to polyvinylidene difluoride membranes. The membranes were blocked in TBS containing $0.05 \%$ Tween-20 (TBST) and 5\% nonfat powdered milk and then probed with different primary and secondary antibodies. Proteins were detected by using enhanced chemiluminescence reagents (Thermo, USA). The primary antibodies included anti-VEGFR, anti-p-VEGFR, anti-p38 MAPK, anti-p-p38 MAPK, anti-ERK1/2, anti-p-ERK1/2, anti-AKT, anti-p-AKT, anti-NF- $\kappa B$, anti-p-NF-кB (Cell Signaling Technology, USA), anti-CXCR4, anti-MMP2, anti-MMP9 (Epitomics, USA), anti-mTOR, anti-p-mTOR (Proteintech, USA), and anti-GAPDH (Proteintech, USA) antibodies.

\subsection{Statistical analysis}

All values were represented as means \pm standard error of the mean (SEM). Statistical significance of the data compared with the untreated control was determined using the Student unpaired t-test. Results were considered statistically significant if the $\mathrm{P}$ value was less than 0.05 .

\section{Results}

3.1 RLTS suppressed the growth of mouse tumor in vivo

The effect of RLTS on tumor growth in vivo was assessed by mouse liver cancer cells H22 transplantation tumor models. Compared with the untreated group, treatment with RLTS resulted in significant reduction of tumor weight (The mean tumor weight were $0.53 \mathrm{~g}$ in the untreated group and $0.21 \mathrm{~g}$ in the treated group, respectively), inhibiting tumor growth at a rate of $60.38 \%$ at $100 \mathrm{mg} / \mathrm{kg}$ (Table. 1). No significant body weight loss and any other abnormities were observed in the RLTS-treated mice. 
3.2 RLTS inhibited proliferation and colony formation both in lung and liver cancer cells

The anti-tumor activity of RLTS on lung and liver cancer cells was examined by MTT assay. As shown in Figure 1A and B, stimulation of A549, NCI-H1299, NCI-H460 lung cancer cells with 40, 80, 160, 320, 640, $1280 \mu \mathrm{g} / \mathrm{mL}$ RLTS and BEL-7402, HepG2, SMMC-7721 liver cancer cells with 25, 50, 100, 200, 400, $800 \mu \mathrm{g} / \mathrm{mL}$ RLTS for $48 \mathrm{~h}$, RLTS inhibited these cells viability in a concentration dependent manner. The 50\%-growth inhibitory concentrations (IC50) of RLTS at 48 h was 134, 162, 76, 159, 406 and $61 \mu \mathrm{g} / \mathrm{mL}$, respectively, whereas it was considerably less active in L-02 cells (IC50 was $790 \mu \mathrm{g} / \mathrm{mL}$ ). In addition, the colony formation assay exhibited that RLTS significantly decreased numbers of colonies of NCI-H460 and SMMC-7721 cells in comparison with the control (Figure 1C). These findings indicated that RLTS had potential anti-tumor properties in both lung and liver cancer in vitro.

3.3 RLTS inhibited the migration of NCI-H460 and SMMC-7721 cells

Scratch motility (wound-healing) assay was performed to investigate the effect of RLTS on cell migration. Confluent monolayers of cells were scratched to form wound and cultured in the absence or presence of various concentrations of RLTS for $24 \mathrm{~h}$. as shown in Figure 2, RLTS treated cells moved slowly compared to control cells, suggested that treatment of RLTS suppressed migration of both NCI-H460 and SMMC-7721 cells.

3.4 RLTS induced cell apoptosis in tumor cells

To further demonstrate the anti-cancer effects of RLTS, we investigated the effect of RLTS on cell apoptosis, RLTS treated NCI-H460 cells were stained with Annexin V-FITC and PI and analyzed by flow cytometry. We observed an increase in the percentage of apoptotic cells in the treatment groups (Figure 3). The percentage of 
apoptotic NCI-H460 cells resulting from treatment with $0,17.5,35$ and $70 \mu \mathrm{g} / \mathrm{mL}$ of RLTS for $48 \mathrm{~h}$ was $5.82 \%, 5.30 \%, 9.10 \%$, and $29.83 \%$, respectively.

\subsection{RLTS inhibited PI3K/AKT/mTOR signaling cascades}

In order to investigate the role of RLTS in mediating PI3K/AKT/mTOR activation in tumor cells, the expression of AKT, p-AKT, mTOR and p-mTOR in NCI-H460 and SMMC-7721 cells were analyzed by western blotting. As shown in Figure 4, significantly activation of AKT and mTOR was suppressed in the two cells upon RLTS treatment. Furthermore, activated Akt can subsequently regulate the NF- $\kappa$ B pathway, we also found p-NF-kB p65 was decreased in both NCI-H460 and SMMC-7721 cells. Our data clearly indicated that the inhibition of PI3K/AKT/mTOR and AKT/NF- $\kappa \mathrm{B}$ signaling cascade by RLTS led to the suppression of cell proliferation in NCI-460 and SMMC-7721 cells.

\subsection{RLTS regulated p38 activation in tumor cells}

We next performed western blot analysis to determine whether RLTS regulated the activity of MAPK cascades including ERK1/2 MAPK, and p38 MAPK in tumor cells. As shown in Figure 5, RLTS substantially induced the activation of p38 MAPK, and had no significant effect on the phosphorylation of ERK1/2 in NCI-460 and SMMC-7721 cells.

\subsection{RLTS suppressed activated VEGFR in tumor cells}

The PI3K/Akt cascade could be activated by receptor tyrosine kinases, we next examined the effects of RLTS on the activated VEGFR to investigate whether RLTS suppressed AKT activation by downregulation of VEGFR activity. We found that RLTS suppressed the expression of p-VEGFR in NCI-460 and SMMC-7721 cells (Figure 6).

3.8 RLTS downregulated expression of proteins involved in metastasis 
Additional, we examined the effects of RLTS on the expression of proteins involved in metastasis. These results clearly demonstrated that RLTS also suppressed proteins linked with migration and metastasis, including CXCR4, MMP-2 and MMP-9 (Figure 7).

\section{Discussion}

It had been documented that several triterpenoid saponins had been found in Radix et Rhizoma Leonticis, whose aglycone included oleanolic acid, echinocystic acid, hederagenin and cauloside etc. (Strigina LI et al., 1970; Strigina LI et al., 1974; Strigina LI et al., 1975; Strigina LI et al., 1976; Chetyrina and Kalinovskii, 1979; Yang et al., 2013). Even though RLTS has been known to exert anti-bacteria, analgesic effect and anti-inflammatory effects (Yang et al., 2013), the activity and mechanism of action of RLTS on cancer has not yet been elucidated. The aim of our study was to investigate the effects of RLTS on the PI3K/AKT/mTOR and MAPKs signaling and cellular responses. Here, we found that RLTS not only exerted significant anti-proliferative and pro-apoptotic effects, but also regulated PI3K/AKT/mTOR and p38 MAPK activation in human hepatocellular carcinoma and lung cancer cells.

In our experiment in vivo, murine hepatocellular carcinoma $\mathrm{H} 22$ cell-bearing mice model indicated that RLTS effectively inhibited tumor growth through oral administration. So, we hypothesized it could exhibit potent inhibitory effects in human cancer cells, in vitro experiment showed that RLTS exhibited obvious inhibition on proliferation and colony formation of human hepatocellular carcinoma SMMC-7721 cells and lung cancer NCI-H460 cells but was considerably less active in normal liver cells. RLTS also had potential to suppress cancer cell migration and induce cell apoptosis. 
The phosphatidylinositol 3-kinase (PI3K) /AKT/mammalian target of rapamycin (mTOR) pathway has been reported to be a promising target due to its frequent dysregulation in lung and hepatocellular carcinoma (Fumarola et al., 2014; Grabinski et al., 2012). This is major antiapoptotic/prosurvival pathways that control cell proliferation, survival and malignant transformation (Nissim, 2005; Polivka and Janku, 2014; Karar and Maity; 2011). Our findings more specifically indicated that AKT activation was abrogated by RLTS, which further indicated the possibility that RLTS could interfere with mTOR. As was expected, RLTS suppressed activated mTOR in NCI-460 and SMMC-7721 cells.

$\mathrm{NF}-\kappa \mathrm{B}$ is a major sensor of cell stress, which is known to regulate the expression of various cell survival, proliferative, metastatic, and angiogenic gene products (DiDonato et al., 2012; Luedde and Schwabe, 2011). NF- $\kappa B$ is not a molecule within the core PI3K/Akt/mTOR pathway, but it is downstream of Akt. Activation through Akt is important for many tumorigenic functions of NF-kB. Since, both mTOR and $\mathrm{NF}-\kappa \mathrm{B}$ are downstream effector Akt, it appears that the signaling through Akt can potentially proceed in two mutually exclusive independent directions, either through mTOR or through NF-кB (Romashkova and Makarov, 1999; Salminen and Kaarniranta, 2010). We speculated NF- $\kappa B$ perhaps was suppressed as AKT decrease. Consistent with our hypothesis, NF- $\kappa \mathrm{B}$ activation was downregulated in RLTS treated cells. p38 MAPK has recently gained attention as a tumor suppressor, and p38 activation can induce cell apoptosis and suppress oncogenesis (Bradham and McClay, 2006). The induction of $\mathrm{p} 38$ MAPK activation by RLTS could contribute to the suppression of tumorigenesis in upon RLTS treatment in tumor cells. VEGFR was the upstream molecule of AKT, and we found that VEGFR protein activity in cancer cells were decreased after treatment with RLTS. 
Metastases, rather than primary tumors, are responsible for most cancer deaths, cancer 


\section{Acknowledgments}

This work was supported by National Natural Science Foundation of China (Grant No.

81302800 and 81370088), the National Science Foundation for Post-doctoral

Scientists of China (Grant No. 2013M532062), the Fundamental Research Funds for

the Central Universities, and supporting plan of education ministry's new century

excellent talents (NCET-13-0467). 


\section{References}

1. Bradham C., and D.R. McClay. p38 MAPK in development and cancer. Cell Cycle 5:824-8, 2006.

2. Chetyrina N.S. and A.I. Kalinovskii. Triterpene glycosides of Caulophyllum robustum. The structures of caulosides B and C. Chem. Nat. Compd. 15:146-8, 1979.

3. DiDonato J.A., F. Mercurio and M. Karin. NF-אB and the link between inflammation and cancer. Immunol. Rev.246: 379-400, 2012.

4. Fumarola C., M.A. Bonelli, P.G. Petronini and R.R.Alfieri. Targeting PI3K/AKT/mTOR pathway in non small cell lung cancer. Biochem. Pharmacol. 90:197-207, 2014.

5. Furusato B., A. Mohamed, M. Uhlén and J.S. Rhim. CXCR4 and cancer. Pathol. Int. 60: 497-505, 2010.

6. Gil EMC. Targeting the PI3K/AKT/mTOR pathway in estrogen receptor-positive breast cancer. Cancer. Treat. Rev. 40:862-71, 2014.

7. Grabinski N., F. Ewald, B.T. Hofmann, K. Staufer, U. Schumacher, B. Nashan and M. Jücker. Combined targeting of AKT and mTOR synergistically inhibits proliferation of hepatocellular carcinoma cells. Mol. Cancer. 11:85, 2012.

8. Karar J. and A. Maity. PI3K/AKT/mTOR pathway in angiogenesis. Front. Mol. Neurosci. 4:e51, 2011.

9. Luedde T. and R.F. Schwabe. NF-кB in the liver-linking injury, fibrosis and hepatocellular carcinoma. Nat. Rev. Gastro. Hepat. 8:108-118, 2011.

10. Mook O.R., W.M. Frederiks and C.J. Van Noorden. The role of gelatinases in colorectal cancer progression and metastasis. Biochim. Biophys. Acta. 1705: 69-89. 2004. 
11. Nam K.S. and Y.H.Shon. Suppression of metastasis of human breast cancer cells by chitosan oligosaccharides. J. Microbiol. Biotechnol. 19:629-33, 2009.

12. Nissim H.. The Akt-mTOR tango and its relevance to cancer. Cancer Cell. 8:179-83, 2005.

13. Polivka Jr. J. and F. Janku. Molecular targets for cancer therapy in the PI3K/AKT/mTOR pathway. Pharmacol. Therapeut. 142:164-75, 2014.

14. Romashkova J.A. and S.S. Makarov. NF-kappaB is a target of AKT in anti-apoptotic PDGF signaling. Nature. 401:86-90, 1999.

15. Salminen A. and K. Kaarniranta. Insulin/IGF-1 paradox of aging: regulation via AKT/IKK/NF-kappaB signaling. Cell. Signal. 22:573-7, 2010.

16. Slomovitz B.M. and R.L.Coleman. The PI3K/AKT/mTOR pathway as a therapeutic target in endometrial cancer. Clin. Cancer. Res. 18:5856-64, 2012.

17. Strigina L.I., N.S. Chetyrina and G.B.Elyakov. Triterpene glycosides of Caulophyllum robustum. Chem. Nat. Compd. 6:569-71, 1970.

18. Strigina L.I., N.S. Chetyrina, V.V. Isakov, A.K. Dzizenko and G.B. Elyakov. Caulophyllogenin: A novel triterpenoid from roots of Caulophyllum robustum. Phytochemistry. 13:479-80, 1974.

19. Strigina L.I., N.S. Chetyrina, V.V. Isakov, Yu.N. Elkin, A.K. Dzizenko and G.B. Elyakov. Cauloside D a new triterpenoid glycoside from Caulophyllum robustum maxim: Identification of cauloside A. Phytochemistry. 14:1583-6, 1975.

20. Strigina L.I., N.S. Chetyrina and V.V. Isakov. Cauloside G - A new triterpene glycoside from Caulophyllum robustum identification of cauloside C. Chem. Nat. Compd. 12, :553-556, 1976. 
21. Trinh X.B., W.A.A. Tjalma, P.B. Vermeulen, G. Van den Eynden, I. Van der Auwera, S.J. Van Laere, J. Helleman, E.M. Berns, L.Y. Dirix and P.A. van Dam. The VEGF pathway and the AKT/mTOR/p70S6K1 signalling pathway in human epithelial ovarian cancer. Brit. J. Cancer. 100:971-8, 2009 .

22. Vihinen P., R. Ala-aho and V.M. Kahari. Matrix metalloproteinases as therapeutic targets in cancer. Curr. Cancer. Drug. Targets. 5: 203-20, 2005.

23. Wagner E.F. and Á.R. Nebreda. Signal integration by JNK and p38 MAPK pathways in cancer development. Nat. Rev. Cancer. 9:537-549, 2009.

24. Yang X.F., Y.M. Ma, H. Xing, J.J. Liu and Y.X. Kang. Studies on triterpene saponins and their biological activity of Caulophyllum robustum. Journal Shaannxi University Science Technology. 31:62-69, 2013.

25. Yodkeeree S., W. Chaiwangyen, S. Garbisa and P. Limtrakul. Curcumin, demethoxycurcumin and bisdemethoxycurcumin differentially inhibit cancer cell invasion through the down-regulation of MMPs and uPA. J. Nutr. Biochem. 20: 87-95, 2009.

26. Zhang D.M., J.S. Liu, L.J. Deng, M.F. Chen, A. Yiu, H.H. Cao, H.Y. Tian, K.P. Fung, H. Kurihara, J.X. Pan and W.C. Ye. Arenobufagin, a natural bufadienolide from toad venom, induces apoptosis and autophagy in human hepatocellular carcinoma cells through inhibition of PI3K/Akt/mTOR pathway. Carcinogenesis. 34:1331-42, 2013.

27. Zhang Y., L. He, L. Meng and W. Luo. Taspine isolated from Radix et Rhizoma Leonticis inhibits proliferation and migration of endothelial cells as well as chicken chorioallantoic membrane neovascularisation. Vasc. Pharmacol. 48:129-37, 2008.

28. Zhang H., G. Cicchetti, H. Onda, H.B. Koon, K. Asrican, N. Bajraszewski, F. Vazquez, C.L. 
Carpenter and D.J. Kwiatkowski. Loss of Tsc1/Tsc2 activates mTOR and disrupts PI3K-Akt signaling through downregulation of PDGFR. J. Clin. Invest. 112:1223-33, 2003. 


\section{Figure legends}

Figure 1 RLTS suppressed tumor cell proliferation and colony formation. A and B, Effect of RLTS on proliferation of hepatocellular carcinoma cells and lung cancers cells. Cells were treated by RLTS at indicated concentrations for $48 \mathrm{~h}$. The cell proliferation was measured using the MTT assay. Data were represented as the means \pm SEM from five repeated experiments. C, Effect on colony formation of SMMC-7721 and NCI-H460 cells by RLTS. Data were represented as the means \pm SEM from three repeated experiments.

Figure 2 RLTS inhibited migration of tumor cells. A and B, Photographs of wound of cells treated with RLTS. Confluent monolayers of SMMC-7721 (A) and NCI-H460 (B) cells were scarred and pretreatment with RLTS for $24 \mathrm{~h}$, the migrating cells into the wound surface were photographed. $\mathrm{C}$, quantification of $\mathrm{A}$ and $\mathrm{B}$. The results shown were representative of three independent experiments. Data were expressed as mean \pm SEM. $* \mathrm{P}<0.05, * * \mathrm{P}<0.01$ compared with untreated control cells.

Figure 3 RLTS induced cell apoptosis in tumor cells. NCI-H460 cells were treated with indicated concentrations of RLTS for $48 \mathrm{~h}$ and the cells were incubated with an Annexin V-FITC and PI and then analyzed by a flow cytometry.

Figure 4 RLTS suppressed PI3K/AKT/mTOR signaling pathway in tumor cells. A, SMMC-7721 and NCI-H460 cells were treated with various concentrations of RLTS for $48 \mathrm{~h}$. Then equal amounts of lysates were analyzed by western blotting using antibodies against p-AKT,

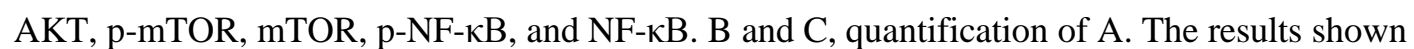
were representative of three independent experiments. Data were expressed as mean $\pm \mathrm{SEM}$. $* \mathrm{P}<$ $0.05, * * \mathrm{P}<0.01$ compared with untreated control cells.

Figure 5 RLTS suppressed p38 MAPK signaling in tumor cells. A, SMMC-7721 and NCI-H460 cells were treated with various indicated concentrations of RLTS for $48 \mathrm{~h}$. Then equal amounts of lysates were analyzed by western blotting using antibodies against p-ERK1/2, ERK1/2, p-p38 MAPK, and p38 MAPK. B and C, quantification of A. The results shown were representative of three independent experiments. Data were expressed as mean \pm SEM. $* \mathrm{P}<0.05, * * \mathrm{P}<0.01$ 
compared with untreated control cells.

Figure 6 RLTS inhibited activated VEGFR expression in cancer cells. A, SMMC-7721and NCI-H460 cells were treated with the indicated concentrations of RLTS for $48 \mathrm{~h}$, then equal amounts of lysates were analyzed by western blotting using individual antibodies.. B, quantification of A. The results shown were representative of three independent experiments. $* \mathrm{P}<$ 0.05 compared with untreated control cells.

Figure 7 RLTS downregulated expression of proteins related to metastasis. A, western blot analysis of CXCR4, MMP-2 and MMP-9 protein expression in NCI-H460 cells after treatment with RLTS at indicated concentrations for $48 \mathrm{~h}$. B, quantification of A. The results shown were representative of three independent experiments. Data were expressed as mean $\pm \mathrm{SEM}$. $* \mathrm{P}<0.05$, **P $<0.01$ compared with untreated control cells. 
A

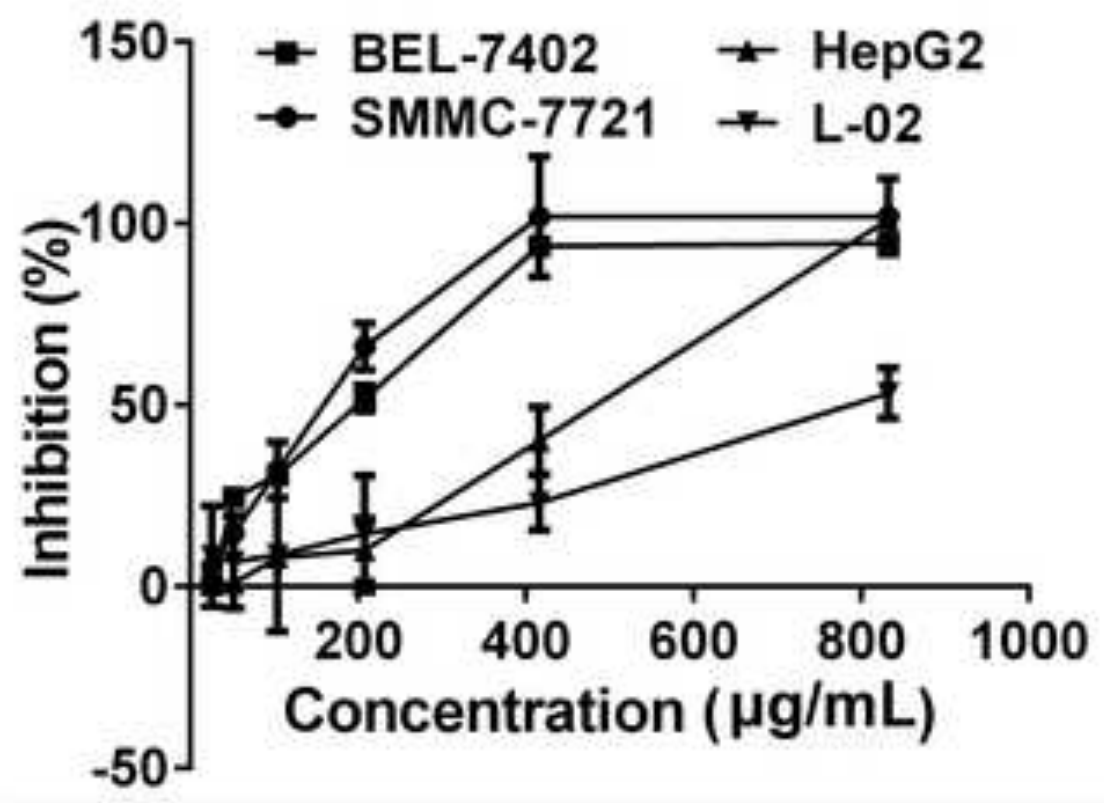

B

C

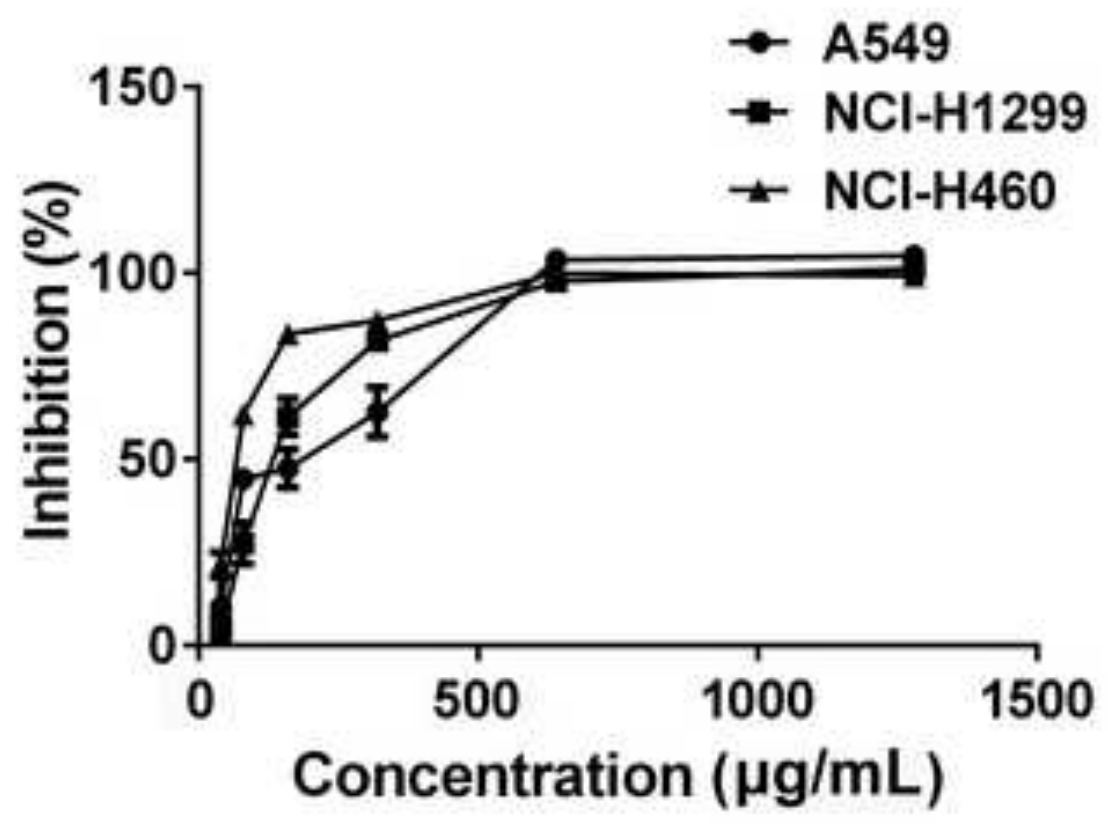

SMMC-7721

$\begin{array}{lllll}0 & 15 & 30 & 60 & \mu \mathrm{g} / \mathrm{mL}\end{array}$

$\mathrm{NCl}-\mathrm{H} 460$

$\begin{array}{lllll}0 & 17.5 & 35 & 70 & \mu \mathrm{g} / \mathrm{mL}\end{array}$


A

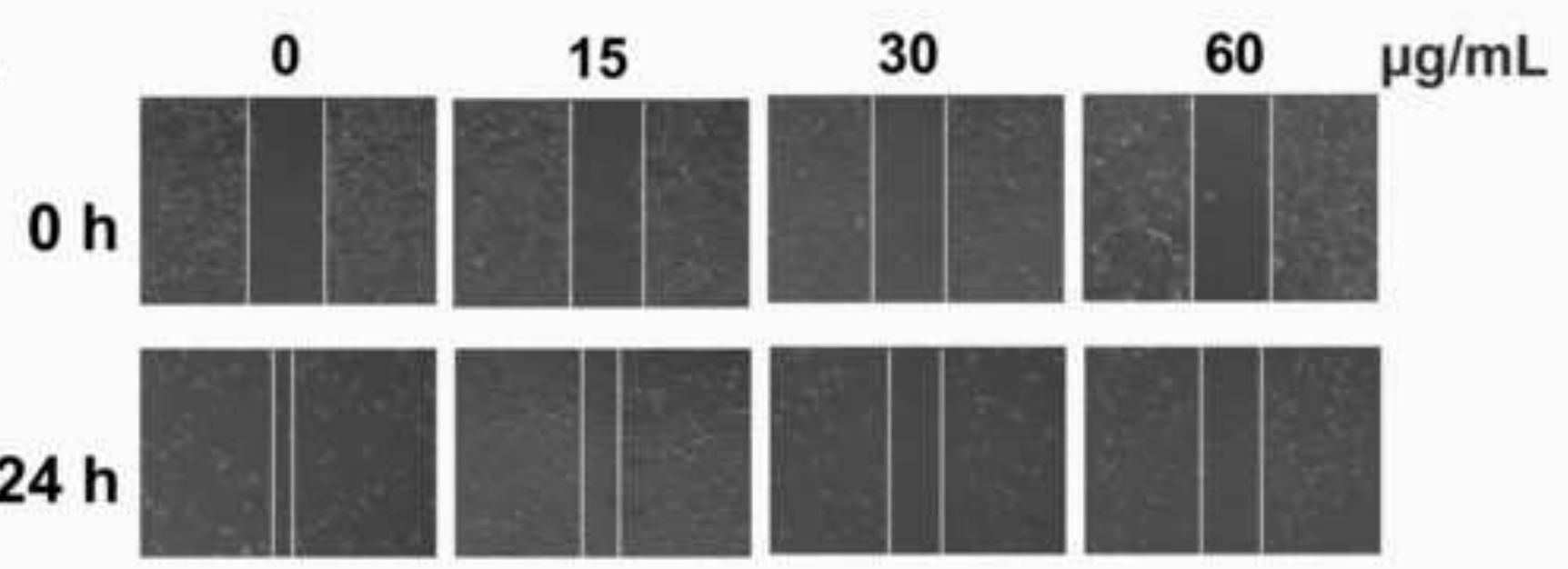

B
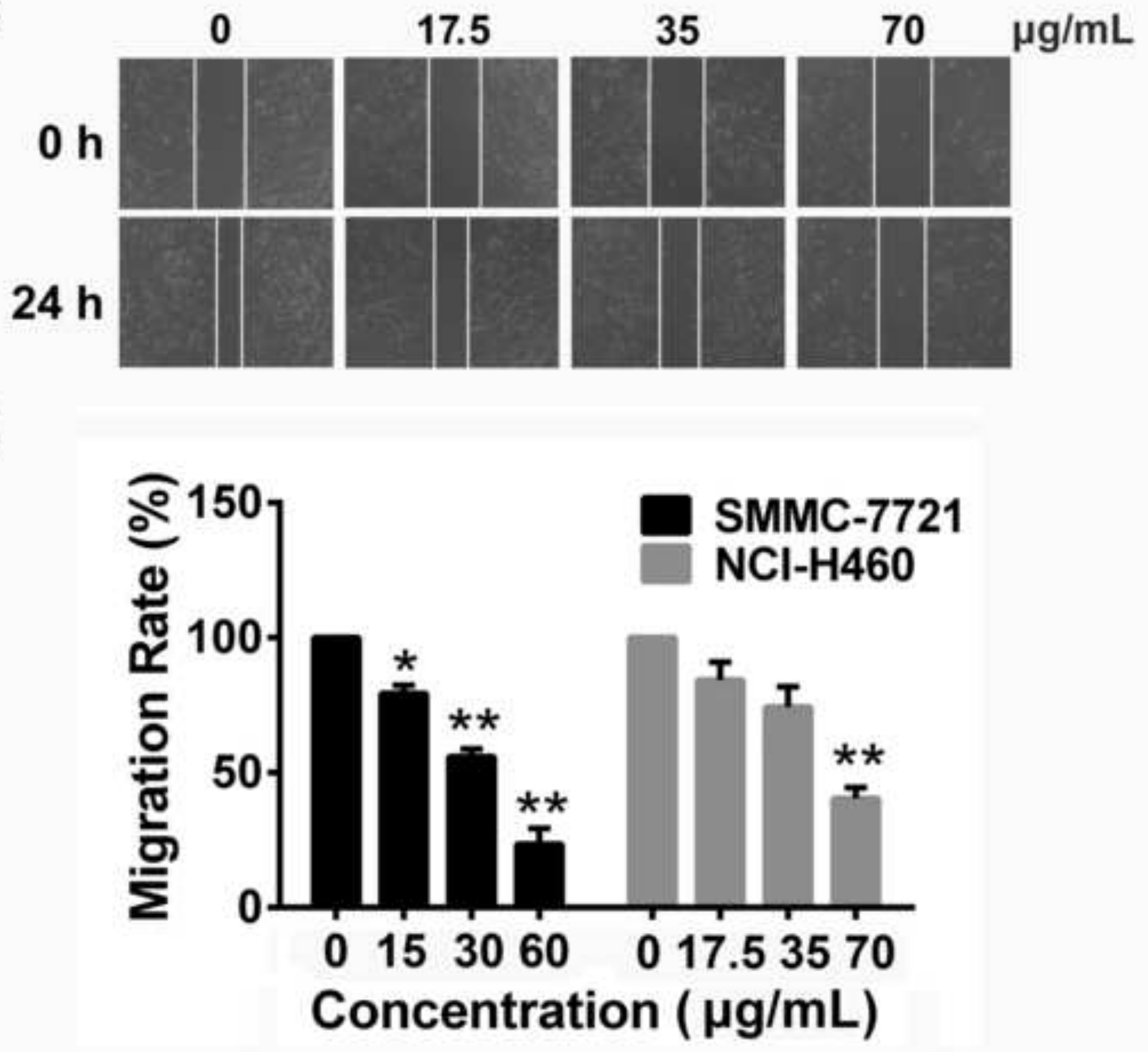

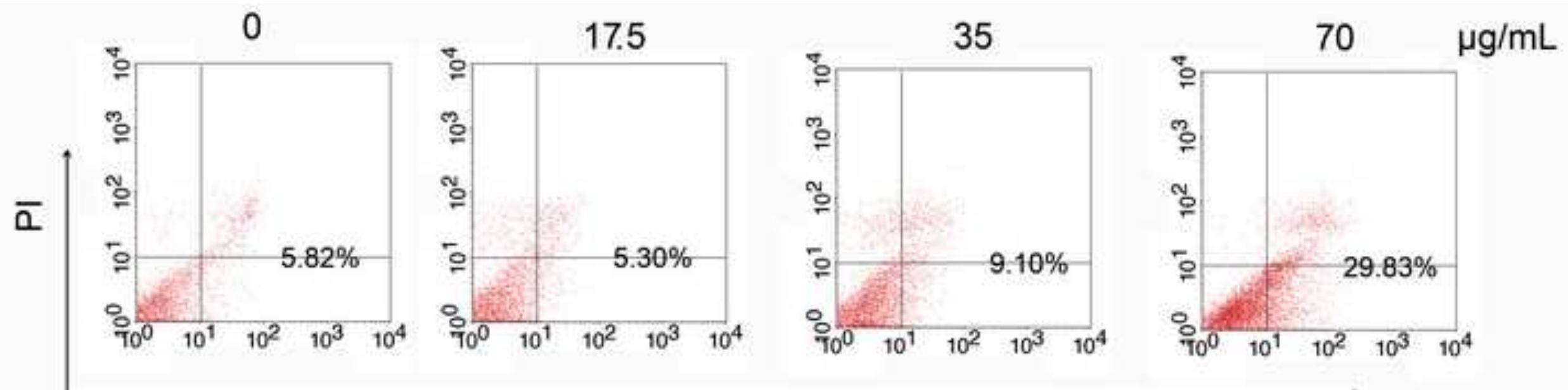

\section{Annexin V FITC}




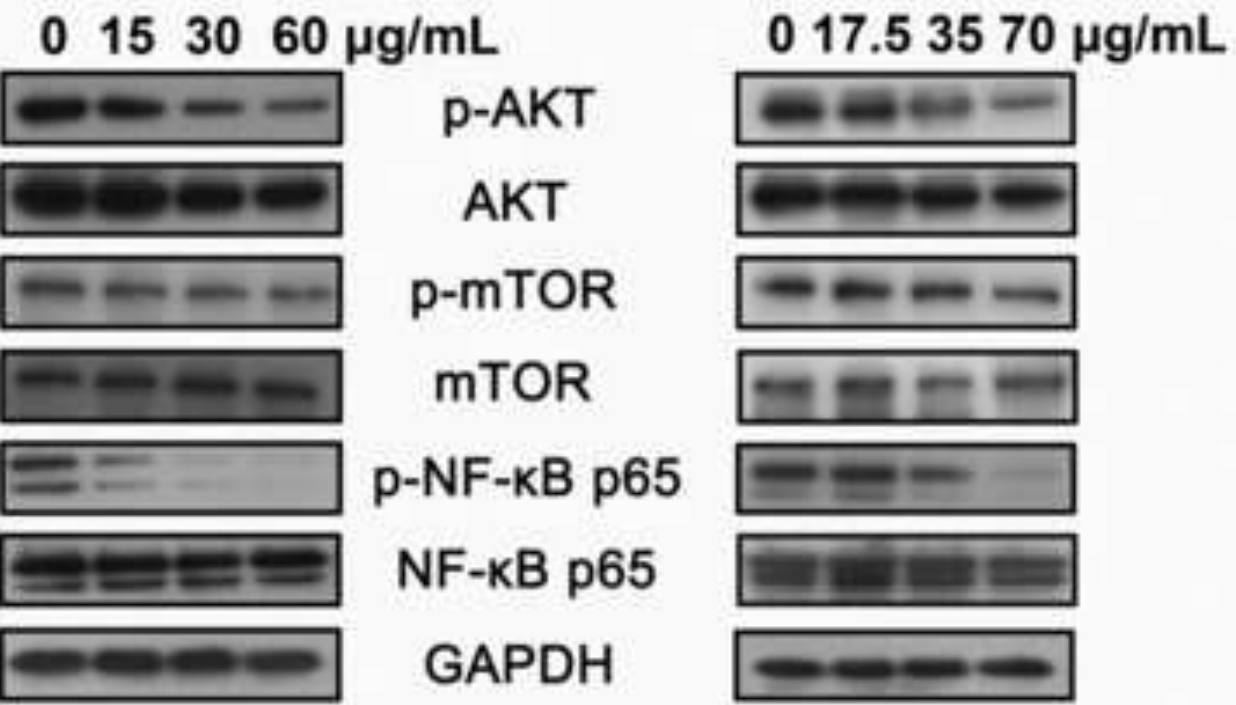

B

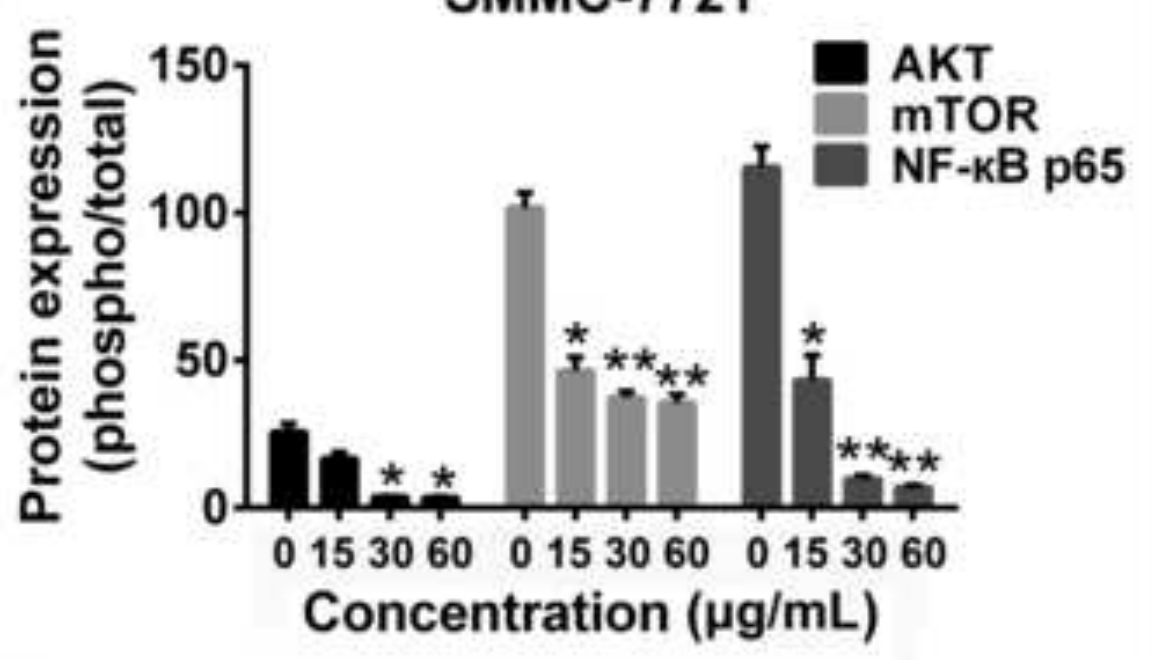

\section{$\mathrm{NCl}-\mathrm{H} 460$}

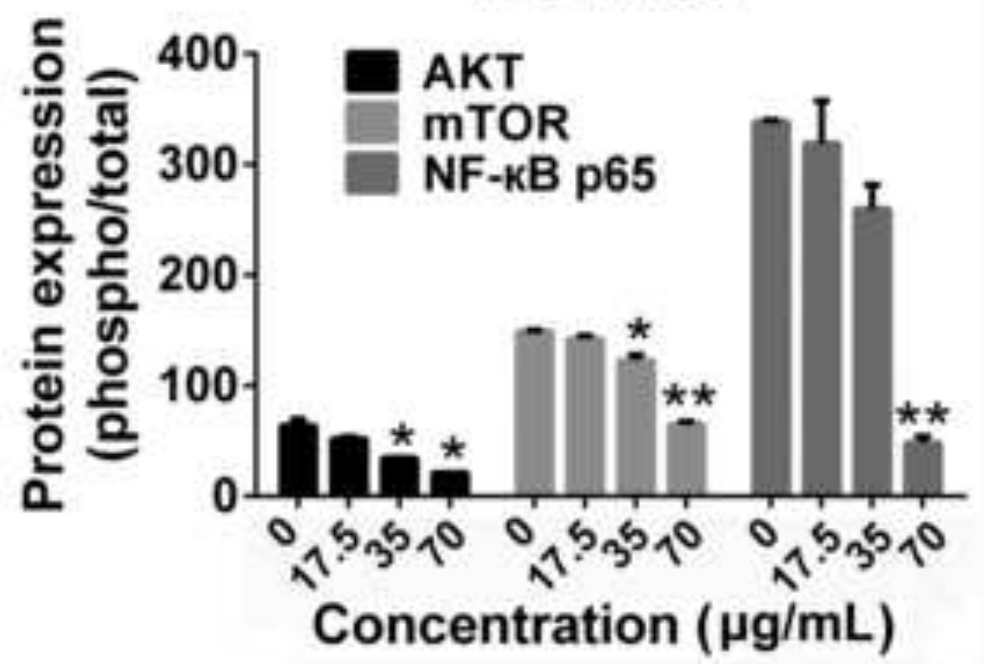


A SMMC-7721

$0153060 \mu \mathrm{g} / \mathrm{mL}$

$\mathrm{NCl}-\mathrm{H} 460$

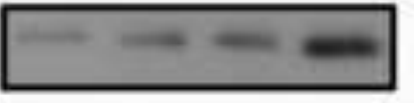

p-p38

$017.53570 \mu \mathrm{g} / \mathrm{mL}$

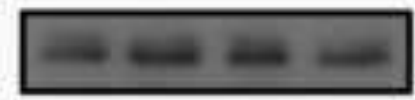

p38

p-ERK $1 / 2$

ERK1/2

GAPDH
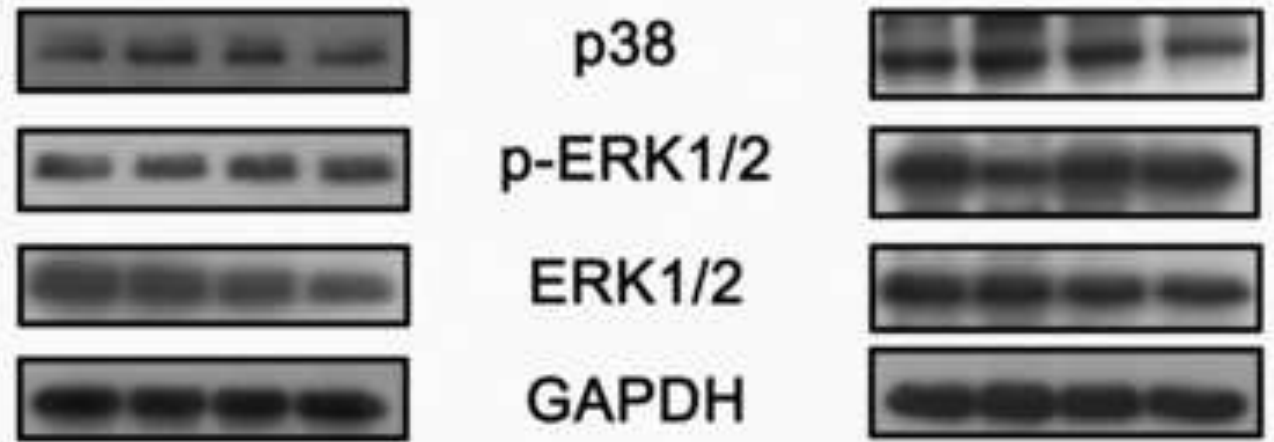

SMMC-7721

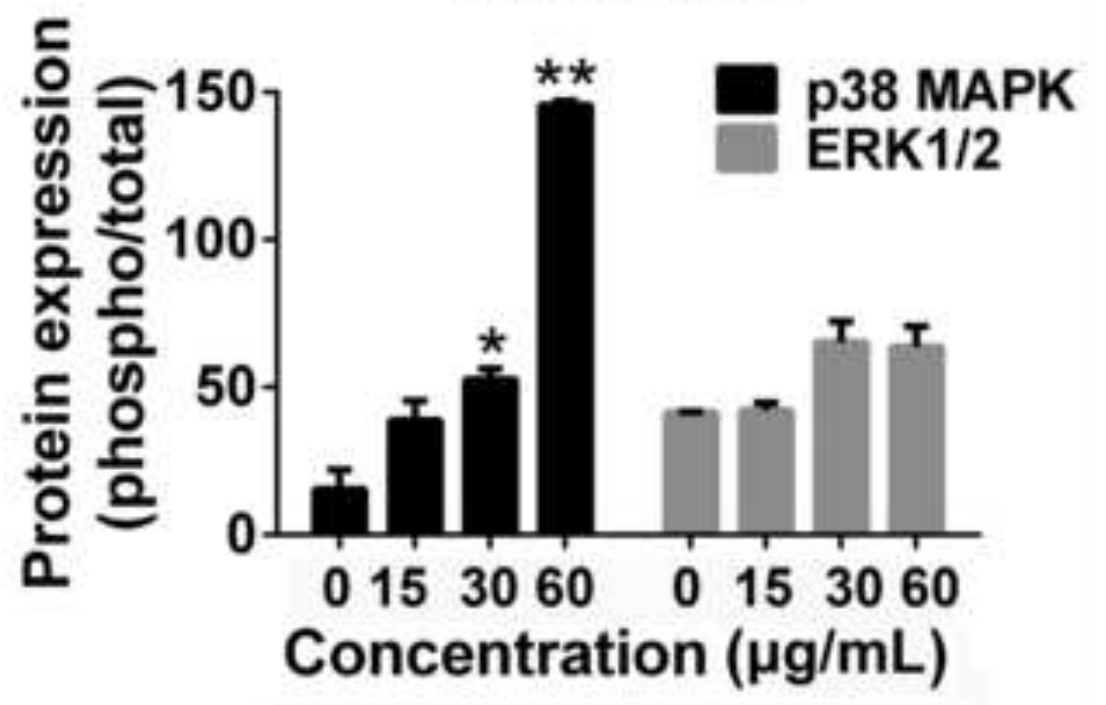

$\mathrm{NCl}-\mathrm{H} 460$

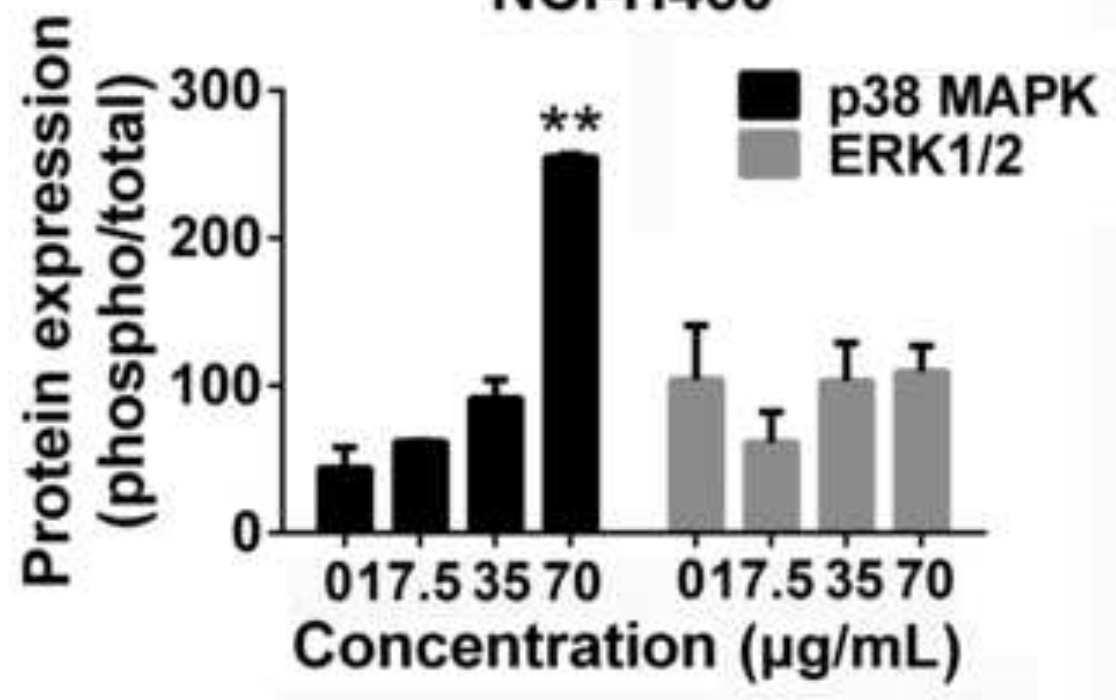


A

SMMC-7721

$\mathrm{NCl}-\mathrm{H} 460$

$0153060 \mu \mathrm{g} / \mathrm{mL}$

p-VEGFR2

VEGFR2

0 $17.53570 \mu \mathrm{g} / \mathrm{mL}$

GAPDH

B

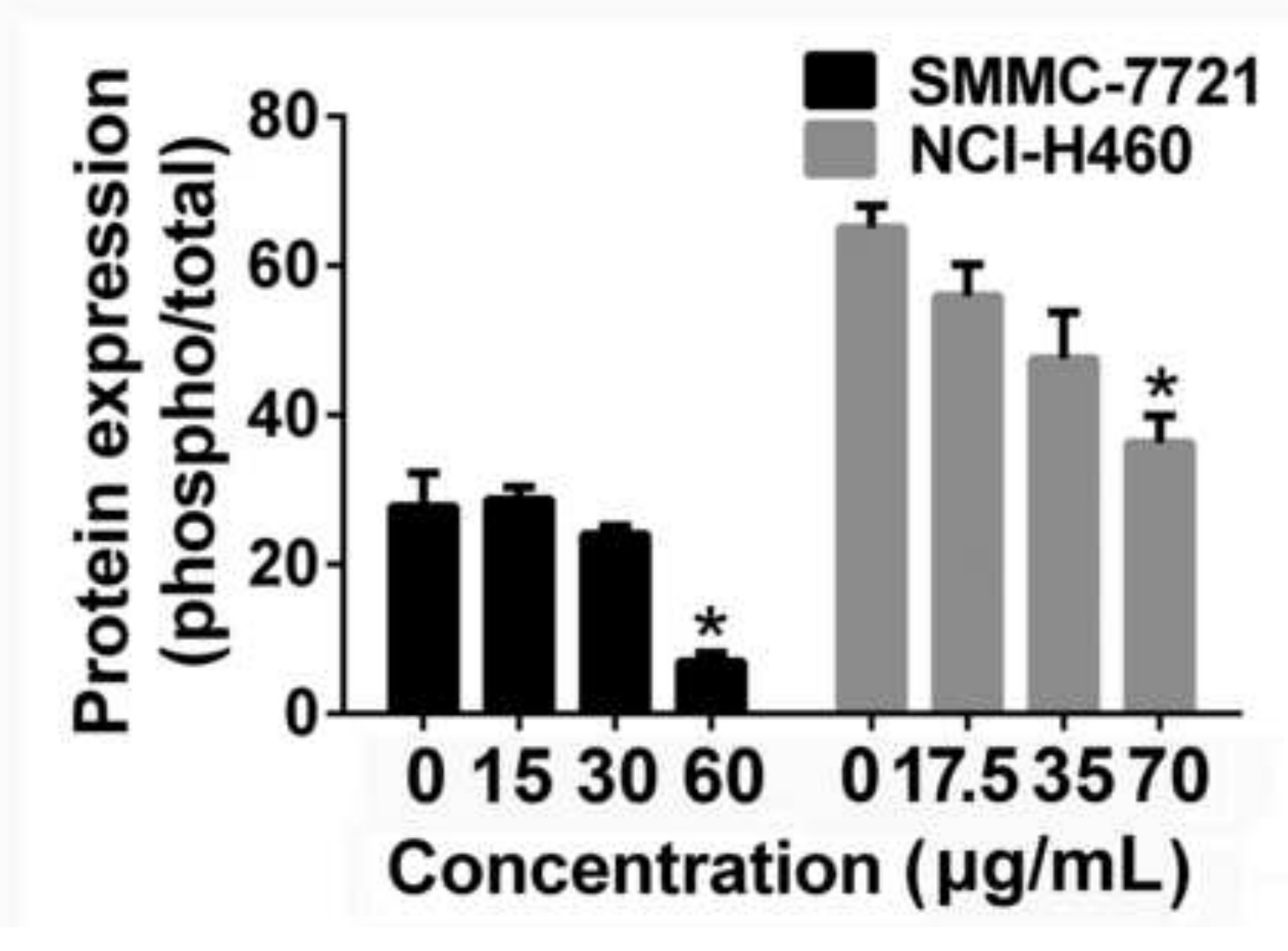

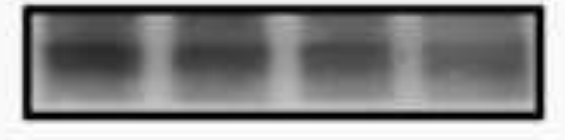
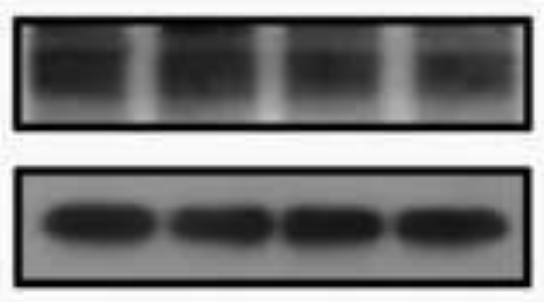

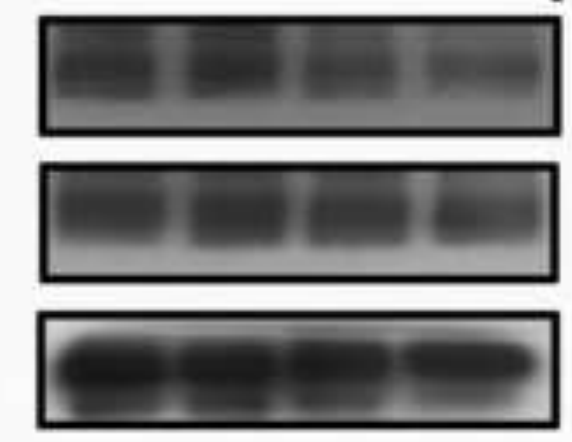




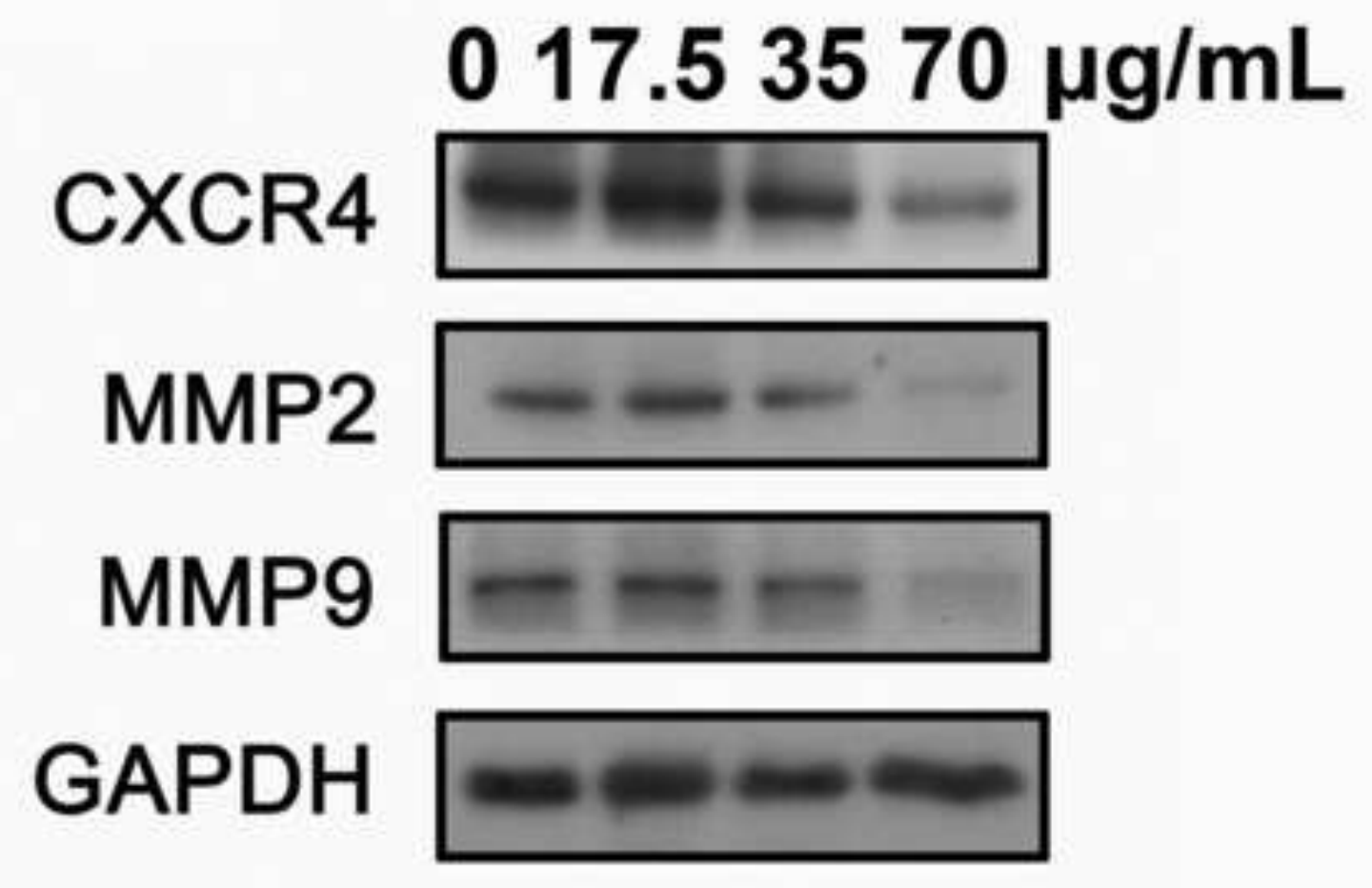

B

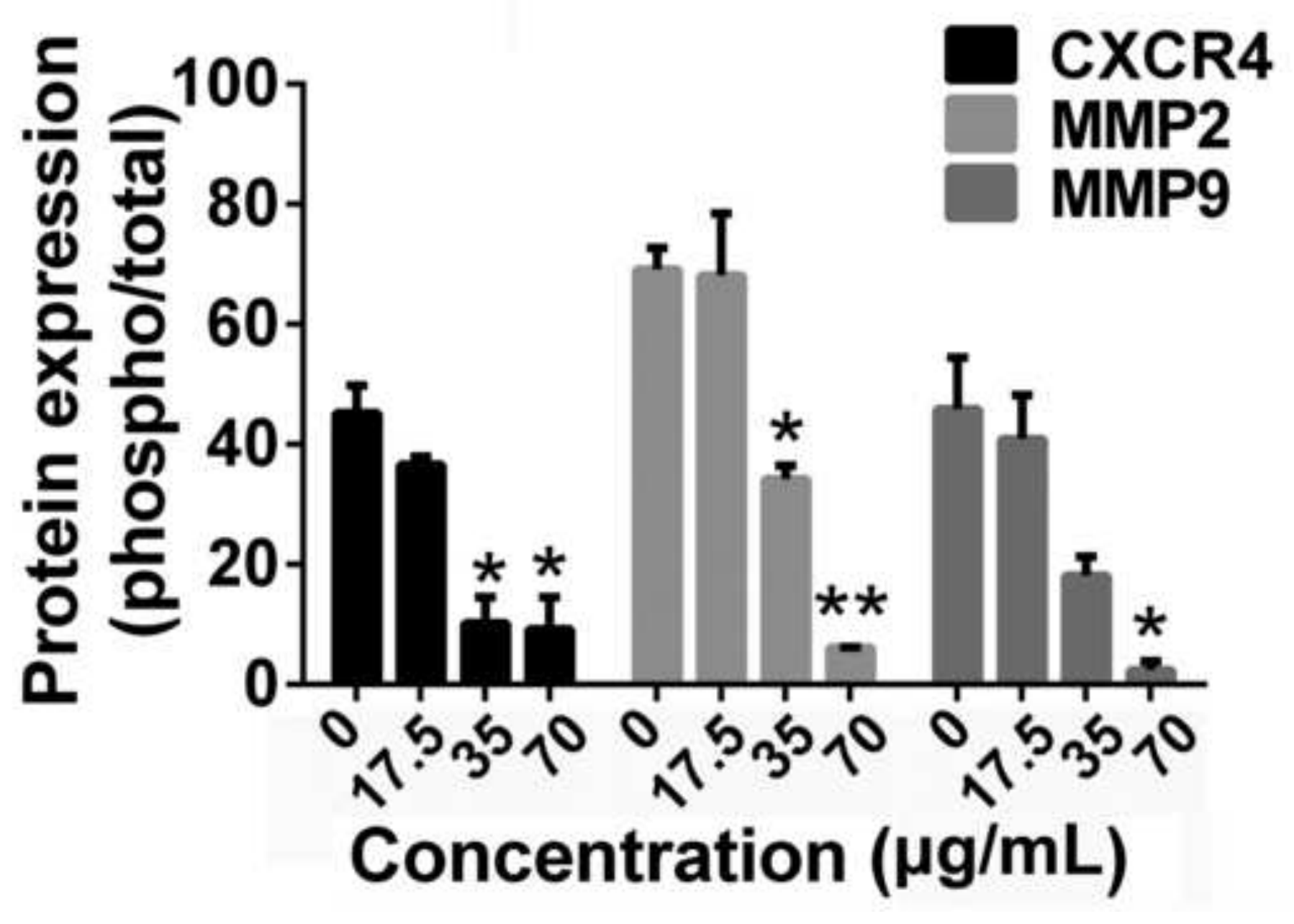

Figure 7

A

\section{$017.53570 \mu \mathrm{g} / \mathrm{mL}$}

CXCR4

MMP2

MMP9

GAPDH

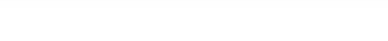
(2)

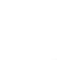

$$
\text { .9. }
$$

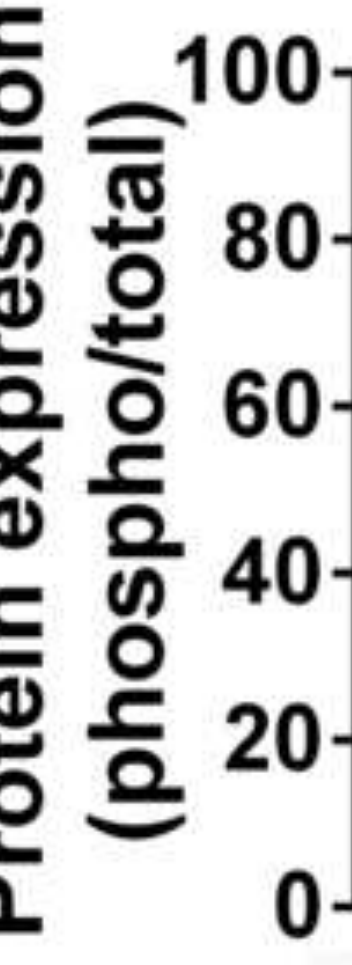$$
\text { Concentration }(\mu \mathrm{g} / \mathrm{mL})
$$ 
Table 1 In vivo inhibitory effect of RLTS on H22 cells

\begin{tabular}{ccc}
\hline Treatment & Control & RLTS \\
\hline Dose $(\mathrm{mg} / \mathrm{kg})$ & - & 100 \\
Initial tumor & $105.45 \pm 21.01$ & $101.18 \pm 16.68$ \\
volume $\left(\mathrm{mm}^{3}\right)$ & \\
$\quad \begin{array}{l}\text { Final tumor } \\
\text { weight }(\mathrm{g})\end{array}$ & $0.53 \pm 0.43$ & $0.21 \pm 0.18$ \\
Tumor growth & & 60.38 \\
inhibition $(\mathrm{g} / \mathrm{g}, \%)$ & & \\
Final body & & $27.87 \pm 1.28$ \\
weight $(\mathrm{g})$ & $28.96 \pm 1.93$ &
\end{tabular}

Values are mean \pm standard error of the mean (SEM). 